\section{Wissenschaft im Dialog - Technikakzeptanz als Ziel?}

\author{
von Dorothee Menhart, Wissenschaft im \\ Dialog (WiD)
}

Die Initiative „Wissenschaft im Dialog“ (WiD) will mit Ausstellungen, wissenschaftlichen Filmfestivals, allgemeinverständlichen Vorträgen und einem interaktiven Webauftritt die Gesellschaft für Wissenschaften begeistern und den Dialog zwischen Forschern und Öffentlichkeit in Deutschland fördern. Die WiD wurde 1999 von den großen deutschen Wissenschaftsorganisationen mit Unterstützung des Stifterverbandes für die Deutsche Wissenschaft und des Bundesministeriums für Bildung und Forschung gegründet. Das Akronym „PUSH“ (Public Understanding of Science and Humanities), das für die Aktivitäten in der Gründungsphase der Initiative zunächst verwendet worden war, wurde mittlerweile aufgegeben: Denn WiD will die Öffentlichkeit nicht zur "Akzeptanz" von Wissenschaft und Technik ,pushen“, sondern sie in einen Dialog ziehen. Insofern ist das Ziel "Technikakzeptanz" bei WiD im Sinne einer Bereitschaft der Gesellschaft zum Dialog zu verstehen.

Seit die Präsidenten und Vorsitzenden aller großen deutschen Wissenschaftsorganisationen am 27. Mai 1999 ein Memorandum unterschrieben, mit dem sie sich zur Förderung des Dialogs von Wissenschaft und Gesellschaft verpflichteten, ist es Aufgabe der Initiative „Wissenschaft im Dialog“ durch ein jährliches Wissenschaftsfestival - den ,Wissenschaftssommer" - sowie durch einen interaktiven Webauftritt den Dialog zwischen Forschern und der an Wissenschaften interessierten Öffentlichkeit zu fördern. Über die Website von WiD können Wissbegierige jederzeit Fragen an Forscher stellen und erfahren dann - vermittelt durch Wissenschaftsjournalisten - per E-Mail oder auf der Website von WiD, wie die Antwort auf ihre jeweilige Frage lautet. Während des ein- bis zweiwöchigen Wissenschaftssommers können Interessierte in Ausstellungen, bei Diskussionsveranstaltungen oder auch in Expertenhearings mit Forschern in Kontakt treten und mit ihnen aktuelle Themen aus der Wissenschaft diskutieren.
Neben WiD gibt es vielerlei kleinere Initiativen, die auf einen Dialog oder ein tieferes Verständnis von Wissenschaften in der Öffentlichkeit hinwirken und zu Teilen lange vor Gründung von WiD aktiv wurden. Zielgruppen sind Kinder (z. B. in den zahlreichen „Kinderuniversitäten“), Schüler (z. B. in den mittlerweile bundesweit an Schulen, Hochschulen, Forschungseinrichtungen sowie von Unternehmen eröffneten „Schülerlaboren“) und Erwachsene, die z. B. zu Vorträgen geladen sind, in denen Wissenschaft und Forschung allgemeinverständlich dargeboten werden.

All diesen Initiativen ist gemein, dass sie indem sie Einblicke in Wissenschaft und Technik gewähren und durch den Dialog zwischen Forschern und Öffentlichkeit deren Verständnis fördern - auch auf eine Akzeptanz von Technik hinwirken. Wissenschaft im Dialog und mit ähnlichem Ziel arbeitende andere Initiativen verfolgen das Ziel der Technikakzeptanz aber im ganz ursprünglichen Sinne von Akzeptieren. Akzeptieren wird verstand als „Annehmen“ im Sinne von „Offenheit entgegenbringen“. Mit unqualifizierter Hinnahmebereitschaft hat diese Akzeptanz von Technik und wissenschaftlichem Fortschritt nichts zu tun. Im Gegenteil: Kritische Akzeptanz ist erwünscht und ist für WiD kein Widerspruch in sich. „Wissenschaft im Dialog will definitiv keine kritiklose Akzeptanz z. B. der Technik, aber ebenso wenig eine kritiklose Verweigerung“", sagt Joachim Treusch, der Vorsitzende des Lenkungsausschusses des WiD. Treusch ist Physiker und Vorstandsvorsitzender des Forschungszentrums Jülich.

Technikkritik gilt der Initiative daher als notwendiger Teil - und nicht als Gegensatz von Technikakzeptanz. Treusch: „Wissenschaft lebt ja geradezu von ihrer Ergebnisoffenheit. Also war es immer das Bemühen von WiD, die Öffentlichkeit so in den Dialog zu ziehen, dass sie gerade für diese beruflich bedingte Notwendigkeit der Vorurteilslosigkeit der Wissenschaft und der Wissenschaftler ein Gefühl bekommt.“

Während der jährlich stattfindenden Wissenschaftssommer, die WiD seit mittlerweile sechs Jahren jedes Jahr in einer anderen Stadt und in einem anderen Bundesland organisiert, steht so auch der Dialog im Vordergrund: Ausstellungsstücke einzelner Forschungsorganisationen stehen nicht einfach nur zur Besichtigung da, sondern werden in Idee und Funkti- 
onsweise von Forschern erläutert und laden außerdem zum Experimentieren, zum Anfassen und Ausprobieren ein. Auch stehen Wissenschaftler bereit, um über ihre Forschung zu erzählen und die Ausstellungsbesucher haben die Chance, tatsächlich einen Einblick in die Welt und Arbeitsweise des Forschens zu bekommen. „Dass wir es dabei nie (oder nur ganz selten) schaffen werden, einen Laien zum wirklichen inhaltlichen Verstehen eines wissenschaftlich komplexen Sachverhaltes zu bringen, spricht so wenig gegen den Dialog, wie die Unfähigkeit, Noten zu lesen, gegen den Besuch einer Mozartoper spricht. Wenn es gelingt, das Verständnis der Öffentlichkeit dafür, was Wissenschaft ist und kann (als ergebnisoffener Prozess, der bei der Zukunftsbewältigung hilft) $\mathrm{zu}$ stärken, und deutlich $\mathrm{zu}$ machen, dass Wissenschaft konstitutiv für unsere aufgeklärte Gesellschaft ist, dann haben wir einiges erreicht", sagt Treusch.

So bedeutet gesellschaftliche Akzeptanz von Wissenschaft und Technik für WiD letztlich die Bereitschaft der Gesellschaft, dem wissenschaftlichen Fortschritt Interesse entgegenzubringen und sich eine möglichst umfassende Meinung zu aktuellen Themen zu bilden. Wird beispielsweise an die rote Gentechnik gedacht, so zeigt sich, dass die aktuellen Themen schließlich jeden Einzelnen betreffen und die Gesellschaft in ihren ethischen und kulturellen Vorstellungen jetzt und auch zukünftig in einem Maße beeinflussen, dass Entscheidungen über bestimmte Entwicklungen auf der Basis eines möglichst breiten gesellschaftlichen Konsenses getroffen werden sollten.

Mit dem schon zu Gründungszeiten und seither immer wieder einmal aufkommenden Vorwurf, der Initiative Wissenschaft im Dialog gehe es bei all ihren Anstrengungen schlicht um Akzeptanzbeschaffung, geht sie gelassen um: Natürlich wollen die hinter der Initiative stehenden Forschungsorganisationen sowie Unterstützer wie das Bundesforschungsministerium und der Stifterverband für die Deutsche Wissenschaft, dass Forschung und Wissenschaft, neue Technologien und innovatives Denken die Aufmerksamkeit in der Gesellschaft erlangen, die sie verdienen. Schon 1999 wurde klar gesagt, dass es, um ,den gesellschaftlichen Stellenwert der Wissenschaften und der Technik deutlich zu machen, (...) öffentlicher Überzeugung mit Hilfe einer intensiven, gezielten und professionell koordinierten Kommunikation" bedürfe (Memorandum 1999).

Die Wissenschaftsorganisationen begreifen ihr Wirken jedoch nicht als PR-Aktion, sondern als Aufklärungsarbeit, auf die die Gesellschaft einen Anspruch hat - wegen des immensen Einflusses wissenschaftlichen Fortschritts auf die Lebensbedingungen der Menschen, auf die kulturelle Entwicklung der Gesellschaft und auch deshalb, weil Wissenschaft und Forschung zum allergrößten Teil mit öffentlichem Geld finanziert werden. So gehen die Unterzeichner des Memorandums davon aus, ,dass man mit immer weniger Bereitschaft seitens der Öffentlichkeit rechnen darf, diejenige Forschung und Entwicklung zu unterstützen, die die Erörterung ihrer gesellschaftlichen Relevanz unter Einbeziehung realistischer Chancen und möglicher Risiken unterlässt. Diese Legitimation kann eingefordert werden, weil hinreichend erklärt und verständlich gemacht werden muss, warum sie mit öffentlichem Geld zu unterstützen ist.“ (Memorandum 1999)

Zudem, so steht es in dem damals verabschiedeten Memorandum, könnten mangelndes Verständnis für Wissenschaft, aber auch enttäuschte Erwartungen und Wunderhoffnungen elementare Folgeprobleme auslösen, die der Gesellschaft schaden und von einer Verweigerungshaltung bis zur Hinwendung zur PseudoWissenschaft reichen. Je stärker aber Wissenschaft und Technik direkt in das Leben der Menschen eingreifen - man denke an die Medizintechnik - und je abhängiger die deutsche Gesellschaft vom „Kapital“ des Wissens wird, desto dringlicher stellt sich für die Akteure die Frage nach der öffentlichen Teilhabe im Sinne eines demokratischen Prozesses. Im „Jahr der Lebenswissenschaften" zum Beispiel wurde während des Wissenschaftssommers in Berlin daher die Stammzellforschung in den Mittelpunkt vieler öffentlicher Diskussionsveranstaltungen gestellt. Die Evangelische Akademie zu Berlin lud die Öffentlichkeit ein, einige der ethischen und gesellschaftspolitischen Fragestellungen aufzugreifen und zu diskutieren, die mit der Entschlüsselung des Humangenoms einhergehen. Das Wissenschaftszentrum Berlin für Sozialforschung (WZB) befasste sich in einem für jeden offenen Workshop mit den möglichen sozialen und kulturellen Folgen der 
Anwendung und Verbreitung humangenetischer Verfahren und in Dresden fand damals organisiert vom Deutschen Hygienemuseum die erste bundesweite Bürgerkonferenz zum Streitfall Gendiagnostik statt.

So geht es der Initiative Wissenschaft im Dialog und anderen am PUSH-Prozess beteiligten Initiativen nicht darum, einer etwaigen Technik-, Wissenschafts- oder Fortschrittsfeindlichkeit entgegenzuwirken, sondern vorausschauend Veränderungen öffentlich zu diskutieren, die individuell spürbar die Bedingungen des Lebens verändern.

Nach Einschätzung des WiD-Geschäftsführers Herbert Münder ist eine tiefgreifende Technikfeindlichkeit in Deutschland ohnehin nicht zu konstatieren, eher eine „Zurückhaltung" innovativer Technik gegenüber, was sich zum Beispiel darin zeige, dass Technik nicht einfach kritiklos übernommen werde wie in Japan: Dort sind schon halbfertige Industrieprodukte auf dem Markt erfolgreich. Auch eine Studie des Büros für Technikfolgen-Abschätzung beim Deutschen Bundestag (TAB) aus dem Jahr 2002 hatte gezeigt, dass sich die Einstellung der Deutschen zu Technik und Wissenschaft mittlerweile deutlich zum Positiven gewandelt hat: Mehr als drei Viertel der Befragten gaben in einer repräsentativen Umfrage im Auftrag des TAB an, dass sie Technik und technischem Fortschritt alles in allem positiv gegenüber stehen. Bei einer früheren Umfrage von 1997 waren es 68 Prozent gewesen. Auch wenn nach wie vor das Thema „Abbau von Arbeitsplätzen durch technische Rationalisierung" von Bedeutung für die Technikeinstellung sei, hätten die anhaltende Wirtschaftskrise und die Diskussionen um die Konkurrenzfähigkeit der deutschen Wirtschaft doch zu einer positiven Veränderung in der Wahrnehmung des Wirtschaftsfaktors „technische Innovation“ beigetragen, so ein Ergebnis der Studie.

Allein durch eine vorsichtige Zurückhaltung gegenüber Technik, wie sie bei manchen neuen Technologien zu beobachten ist, nimmt die Wissenschaft noch keinen Schaden. Es gehört - wie es auch der Philosoph Jürgen Mittelstraß beschreibt - ,eher zu den Stärken einer rationalen Gesellschaft als zu ihren Schwächen, wenn wissenschaftliche Errungenschaften und ihre Anwendungen heute nicht mehr unkritisch akzeptiert werden" (Kegel 2004).
Kritische Vorsicht ist Diskursen nicht abträglich, sondern vielleicht sogar Ansporn für Wissenschaftler, sich ihrer eigenen Forschung und ihres Tuns immer wieder selbst zu vergewissern. Auch, weil sie sich rechtfertigen - oder zumindest erklären - müssen. Denn dass sich nicht nur die Öffentlichkeit der Wissenschaft gegenüber stärker öffnen kann, sondern es auch die Aufgabe von Wissenschaftlern selbst ist, sich der Öffentlichkeit zu stellen, ist für die PUSH-Initiatoren selbstverständlich gewesen. Doch wie Arend Oetker, der Präsident des Stifterverbandes, feststellte, gilt noch immer: $\mathrm{Zu}$ viele Wissenschaftler versuchen, ihre Forschungsinhalte ,angebotsorientiert" weiterzugeben, statt sie mit Bürgern als gleichberechtigten Partnern zu diskutieren. (Oetker 2004)

Die Verfasser des Memorandums „Dialog Wissenschaft und Gesellschaft" hatten 1999 festgestellt, dass in der Wissenschaft im Gegensatz zu den anderen kulturellen Bereichen öffentlich bekannte Leitfiguren fehlen, die die Freude an der Wissenschaft glaubwürdig verkörpern. Wissenschaft komme meist anonym daher. Dass sie von Menschen - mit all ihren persönlichen Stärken und Schwächen - gemacht wird, gehe dabei unter.

Im Rahmen einer weiteren PUSH-Initiative hat die Deutsche Forschungsgemeinschaft (DFG) im September 1999 erstmals den Communicator-Preis ausgeschrieben, der vom Stifterverband finanziert wird. Mehr als $200 \mathrm{Be}-$ werbungen gingen ein. Auserwählt als besonders engagierter Communicator seiner Wissenschaft wurde schließlich Albrecht Beutelspacher, der seit Jahren mit außergewöhnlichen Projekten das Publikum für die abstrakte Wissenschaft der Mathematik begeistert. Im Mittelpunkt steht dabei immer der Alltagsbezug der Mathematik. Das Preisgeld in Höhe von 100.000 Euro nutzte Beutelspacher im Übrigen, um das „Mathematikum“ in Gießen voranzutreiben. Das Mathematikum ist ein MitmachMuseum für die Mathematik. Mittlerweile steht dieses Museum seit drei Jahren und zieht Tag für Tag Besucher in seinen Bann. Mit dem Communicator-Preis wurden in den Folgejahren Wissenschaftler unterschiedlichster Disziplinen ausgezeichnet. Seit 2004 wird auch auf europäischer Ebene ein ganz ähnlicher Preis verliehen: der von der Europäischen Kommission ausgelobte „Descartes-Preis“. 
Der Vorwurf, Wissenschaftler erweckten allzu oft den Eindruck, die Beantwortung von Fragen aus der Gesellschaft sei ihnen lästig und halte nur von der Arbeit ab (Kegel 2004), mag noch viel zu häufig berechtigt sein. Aber ein Wandel ist für alle in den PUSH-Prozess Involvierten spürbar. Mit zunehmendem Selbstverständnis gehen Forscher auf die Öffentlichkeit zu und versuchen, im Gespräch auch auf Sorgen und Ängste einzugehen, die im Zusammenhang mit Forschungsfeldern wie beispielsweise der Gen- oder der Nanotechnologie aufkommen. Im Jahr 2004 hat die Bundesregierung im Rahmen des "Jahres der Technik“ einen Nano-Truck auf Reisen geschickt, der bei Unternehmen, auf Schulhöfen und in den Fußgängerzonen diverser Städte Halt machte. Dort wurde dann über die neuesten Entwicklungen in der Nano-Technologie informiert. Immer waren Forscher anwesend, die mit den Besuchern in den Dialog treten und auf ihre Fragen und etwaige Bedenken eingehen konnten.

Auch auf Seiten der Gesellschaft scheint das Interesse am Dialog mit Wissenschaftlern zu wachsen: Im Jahr 2002 besuchten rund 70.000 Interessierte den Wissenschaftssommer in Bremen, zwei Jahre später waren es in Stuttgart bereits 110.000. In Berlin und Potsdam, wo der Wissenschaftssommer 2005 erstmals zwei Wochen dauerte, besuchten 130.000 Interessierte die Veranstaltungen von WiD. Als Wissenschaftsjahr des Bundesministeriums für Bildung und Forschung (BMBF) stellte das Jahr der Technik 2004 neue Besucherrekorde auf: Mehr als 1,1 Mio. Besucher kamen zu rund 1.100 Veranstaltungen, in denen in ganz Deutschland über Technik informiert und diskutiert wurde. Das Interesse an Wissenschaft, Forschung und Technik ist also da - und die Bereitschaft der Wissenschaftler wächst, diesem Interesse entgegenzukommen.

WiD hat mit seinen Veranstaltungen insbesondere Schülerinnen und Schüler im Blick. Schließlich ist neben dem erwünschten Diskurs über Entwicklungen in den Wissenschaften auch die Nachwuchsförderung und das Werben von Studienanfängern für technischnaturwissenschaftliche Fächer ein wesentlicher Grund für die Aktivitäten von WiD und BMBF: So war das seit dem Jahr der Geowissenschaften jedes Jahr im Sommer über deutsche Wasserstraßen fahrende Ausstellungs- schiff von WiD seit 2002 jeweils unterwegs. Es zeigte Ausstellungen zur Geowissenschaft, zur Chemie, zur Technik und - im Jahr 2005 - zu Albert Einstein und lockte vor allem Schulklassen an Bord. Insgesamt 103.000 Interessierte sahen allein im Einsteinjahr die Ausstellung, also fast 1.000 pro Öffnungstag.

Die Mittel, mit denen der von WiD organisierte Wissenschaftssommer wie auch die vom BMBF ausgerufenen Wissenschaftsjahre ausgestattet werden, sind seit 2000 beträchtlich gestiegen, und die Initiative Wissenschaft im Dialog hat in der Politik augenscheinlich an Bedeutung gewonnen. Eine ähnliche Entwicklung zeigt sich auch auf europäischer Ebene: Im Rahmen von „Science and Society“ wurde im 6. Rahmenplan erstmals Geld für die Vermittlung von Wissenschaften an eine breite Öffentlichkeit zur Verfügung gestellt. Jetzt soll das entsprechende Programm auch im 7. Rahmenplan fortgeführt und wiederum mit EUFinanzmitteln ausgestattet werden. Weiterhin wird im kommenden Jahr in München die zweite Euroscience-Open-Forum-Konferenz (ESOF 2006) stattfinden, die derzeit vom WiDBüro organisiert wird. Ähnlich wie die berühmte Jahrestagung der „American Association for the Advancement of Science" (AAAS) soll die 2004 erstmals in Stockholm ausgetragene Konferenz den Dialog nicht nur zwischen Wissenschaft und Gesellschaft, sondern auch zwischen Forschern verschiedenster europäischer Länder fördern. Und wie auf europäischer, so tut sich auch auf regionaler Ebene einiges: Der Wettbewerb des Stifterverbandes „Stadt der Wissenschaft" lädt Städte ein, sich um Finanzmittel zu bewerben, um ein ganzes Jahr lang Wissenschaft, Forschung und Technologie in den Mittelpunkt des öffentlichen Interesses zu stellen. Möglichst viele Zielgruppen sollen angesprochen werden, um das Interesse breiter Bevölkerungsschichten an der Wissenschaft zu wecken und einen Identifikationswert für die Bürger der Stadt zu schaffen.

Ein Wehmutstropfen freilich bleibt: Bislang ist es nicht gelungen, ein wesentliches Ziel des Memorandums von 1999 tatsächlich durchzusetzen. Dort heißt es: „Es wird ein den einzelnen Institutionen angemessenes Anreizsystem entwickelt, das geeignet ist, Belohnungen für diejenigen Wissenschaftlerinnen und Wissenschaftler in Aussicht zu stellen, die sich aktiv am 
Dialog mit der Öffentlichkeit engagieren. Das Engagement für diesen Dialog darf dem wissenschaftlichen Ruf nicht abträglich sein, es sollte zu einem zusätzlichen Merkmal wissenschaftlicher Reputation werden.“ Abträglich, so darf man nach sechs Jahren PUSH-Aktivitäten in Deutschland getrost feststellen, ist ein solches Engagement wohl nicht mehr. Ausreichend zuträglich jedoch auch noch nicht. So wird nach wie vor zu Recht gemahnt, dass der Dialog zwischen Wissenschaft und Gesellschaft nicht länger eine zusätzliche Aufgabe darstellen sollte, die neben der wissenschaftlichen und technischen Entwicklung herläuft, sondern selbst als Teil dieses Prozesses verstanden werden und bei Begutachtungen, Berufungsverfahren etc. Anerkennung finden, wie dies bereits in einigen skandinavischen Ländern der Fall ist. (vgl. Ahrens-Radlanski, Klein 2004)

\section{Literatur}

Ahrens-Radlanski, H.; Klein, C., 2004: PUSH setzt Impulse für den Dialog, Mit seinem 1999 ausgelobten Aktionsprogramm „PUSH - Dialog Wissenschaft und Gesellschaft" hat der Stifterverband der Initiative „Wissenschaft im Dialog“ einen zusätzlichen Impuls verliehen. In: Wirtschaft und Wissenschaft spezial. Essen: Verwaltungsgesellschaft für Wissenschaftspflege mbH, S. 8-9

Kegel, B., 2004: Zwischen Faszination und Erschrecken. Wissenschaftspublizistik im Informationszeitalter. In: Wirtschaft und Wissenschaft spezial. Essen: Verwaltungsgesellschaft für Wissenschaftspflege mbH, S. 36-39

Memorandum, 1999: PUSH: Dialog Wissenschaft und Gesellschaft Präambel und Memorandum. In: Wirtschaft und Wissenschaft spezial. Essen: Verwaltungsgesellschaft für Wissenschaftspflege $\mathrm{mbH}$, S. 40-41

Oetker, A., 2004: Nicht innehalten. In: Wirtschaft und Wissenschaft spezial. Essen: Verwaltungsgesellschaft für Wissenschaftspflege mbH, S. 2

TAB - Büro für Technikfolgen-Abschätzung beim Deutschen Bundestag, 2002: Positive Veränderungen des Meinungsklimas - konstante Einstellungsmuster. Dritter Sachstandsbericht des Monitoring „Technikakzeptanz und Kontroversen über Technik“. TAB Arbeitsbereicht Nr. 83, Berlin

\section{Kontakt}

Dorothee Menhart, M.A.

Redakteurin

Wissenschaft im Dialog gGmbH

Markgrafenstraße 37, 10117 Berlin

Tel.: +49 (0) 30 - 20634690

E-Mail: Dorothee.Menhart@w-i-d.de

Internet: http://www.wissenschaft-im-dialog.de

\section{$\langle\gg$}

